\title{
Lateral Sinus Augmentation: A Simplified Safer Approach
}

\author{
Douglas F Dompkowski, Gregori M Kurtzman
}

\begin{abstract}
Enlargement of the maxillary sinus often precludes implant placement in the posterior quadrant without augmentation to create increased bone height to stabilize the implant fixtures and provide load handling following restoration. Maxillary sinus augmentation has been a technical challenge due to the potential for tearing of the Schneiderian membrane during lateral window formation. This article will discuss and review unique instrumentation for a safer simpler approach to lateral window maxillary sinus augmentation.
\end{abstract}

Keywords: Maxillary sinus, Sinus lift, Sinus augmentation, Dental implant.

How to cite this article: Dompkowski DF, Kurtzman GM. Lateral Sinus Augmentation: A Simplified Safer Approach. Int J Oral Implantol Clin Res 2013;4(2):83-89.

\section{Source of support: Nil}

Conflict of interest: Dr. Domkowski has lectured for Hiossen and received honoraria.

\section{INTRODUCTION}

A common clinical problem when implants are to be placed into the posterior maxilla when teeth have been extracted at a prior date is loss of osseous height sufficient to place implants. Some patients show resorptive patterns along with sinus enlargement that leave minimal bone height that can accommodate implant usage. Maxillary sinus augmentation with various bone graft material have become routine treatment over the past 18 years. Numerous studies have reported highly successful implant survival rates when placed into an augmented sinus. ${ }^{1-3}$ Transalveolar sinus floor elevation also referred to as subantral augmentation, was first described by Tatum ${ }^{4}$ and later modified by Summers, ${ }^{5-7}$ uses a series of osteotomes with a mallet to create an osteotomy and in-fracturing the sinus floor while elevating the Schneiderian membrane into the sinus cavity. Following manipulation, the membrane is then lifted and the space created in the sinus is augmented with bone particulate graft material increasing the volume and height of bone available for implant placement.

Numerous studies show that when $>5 \mathrm{~mm}$ of residual alveolar bone is present, simultaneous implant placement can be achieving preformed adequate primary stability. ${ }^{6,8,9}$ Yet, when there is less than $5 \mathrm{~mm}$ of residual alveolar bone height, a delayed 2-stage approach has been recommended for improved implant success. ${ }^{10,11}$ The most common complication of the lateral sinus elevation approach is tearing of the Schneiderian membrane which could allow for bacterial contamination or loose particles gaining access to the sinus cavity. A safer, lateral window approach to sinus augmentation procedure will be discussed using safe end specialized drills with vertical stoppers for osseous window formation and subsequent membrane elevation (Lateral Approach Sinus Kit, Hiossen, Philadelphia, PA, USA/ Osstem, Seoul, South Korea).

\section{MATERIALS AND METHODS}

The Lateral Approach Sinus Kit (LAS-Kit) provides 'dome' drills, 'core' drills, metal stoppers, side wall drill and a bone separator tool (Fig. 1).

The dome drill is a unique osseous drill allowing removal of the lateral wall of the maxillary sinus while collecting autogenous bone to be added to the material to be placed into the sinus. Macro- and micro blades provide excellent cutting of the lateral wall without tearing of the sinus membrane. These dome drills available in both 5.0 and $7.0 \mathrm{~mm}$ diameter are run at 1,200 to $1,500 \mathrm{rpm}$ with irrigation in an implant surgical handpiece. Metal depth control stoppers are provided that fit on the dome drills limiting depth of penetration (0.5, 1.0, 1.5, 2.0, 2.5 and 3.0 $\mathrm{mm}$ ) and are used sequentially to safely expose the sinus membrane.

The core drill, also available in 5.0 and $7.0 \mathrm{~mm}$ diameter differs from the dome drill in that the center does not cut, with bone removal resulting in a core of bone being left over the sinus. This boney lid is removed due to its small size and can be ground to be added to the graft material being placed into the sinus. This particular drill follows the same design of the crestal augmentation sinus (CAS) drills and are utilized at 1,200 to 1,500 rpm as are the dome drills. The metal drill stoppers also fit these drills allowing controlled sequential depth preparation. The bone separator tool is utilized to separate the osseous core created from the core drill.

The side wall drill may be used to enlarge the osseous window created by the dome drill if desired. The tip of this drill is smooth and designed to safely push the sinus membrane away from the cutting portion of the drill, which starts $1 \mathrm{~mm}$ from the safe end. Osseous cutting is performed at $1,500 \mathrm{rpm}$ using the side of the rotating drill to enlarge 


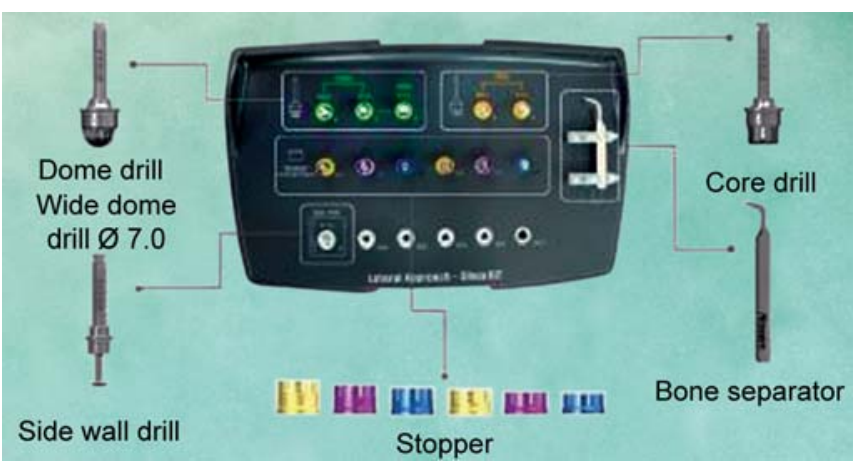

Fig. 1: Lateral approach sinus kit (LAS-Kit)

the osseous window. The metal drill stoppers from the CASKit may be utilized on these side wall drills to limit accidental penetration too far into the sinus and tearing of the membrane during this drills use. As with the other drills in this kit, irrigation is used during its use.

\section{CASE REPORT}

A male aged 65, presented with the desire for rehabilitation of the maxillary arch implant placement with the posterior maxillary left quadrant which had been missing teeth for an extended period of time. Radiographically, enlargement of the maxillary sinus was noted with insufficient height in the molar region for implant placement (Fig. 2). Sinus augmentation was discussed to assist in achieving the patients desired treatment goal. As less than $5 \mathrm{~mm}$ of crestal height was available, implant placement would not be possible at the time of sinus augmentation as primary stability would not be achievable.

Following administration of local anesthetic, a crestal incision with a vertical releasing incision was made and a full thickness flap was elevated (Fig. 2). Elevation of the flap extended superiorly to expose the lateral wall of the maxillary sinus up to the inferior aspect of the zygoma (Fig. 3). Endosseous implants were placed in the posterior right and anterior segments which had sufficient bone height and width for fixture placement.

A $5 \mathrm{~mm}$ wide dome drill was placed onto the surgical handpiece with a $0.5 \mathrm{~mm}$ drill stopper (Fig. 4). This would allow initiation of the window without the possibility of excessive penetration and subsequent damage to the sinus membrane. The dome drill with stopper was placed on the lateral sinus wall at a height more superior then the current height of the available bone as measured radiographically (Fig. 5). This is done to ensure that the window created has elevatable membrane circumferentially. When maximum depth has been achieved with the $0.5 \mathrm{~mm}$ drill stopper present, the drill stopper is changed to a $1.0 \mathrm{~mm}$ stopper and drilling is continued (Fig. 6). The drill stopper is sequentially increased checking for membrane exposure

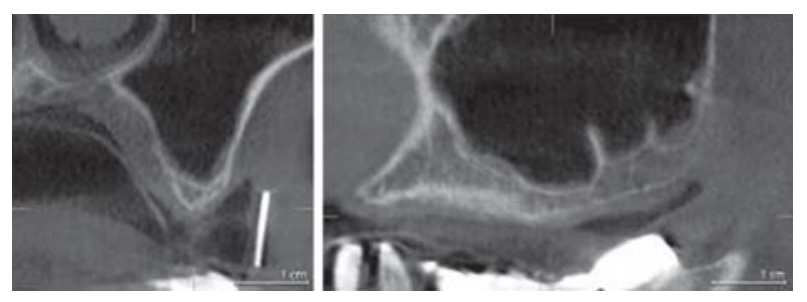

Fig. 2: СBCT pretreatment radiographs demonstrating insufficient osseous height for implant placement without sinus augmentation in the molar region

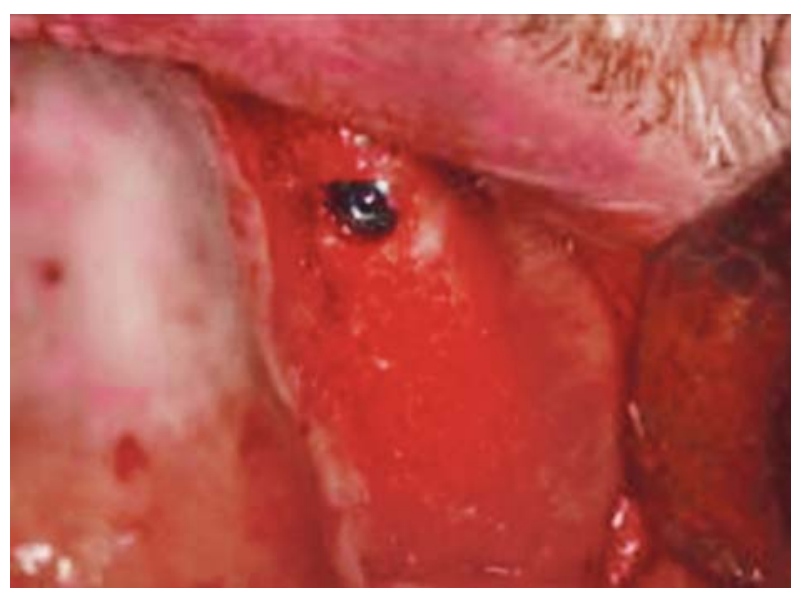

Fig. 3: Lateral aspect of the maxillary posterior following elevation of a full thickness flap

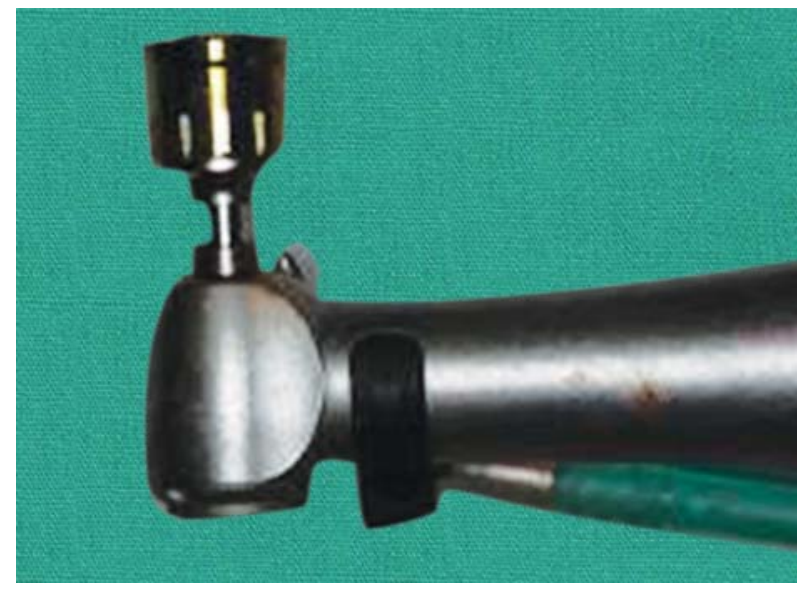

Fig. 4: Dome drill with $0.5 \mathrm{~mm}$ stopper placed on the surgical handpiece

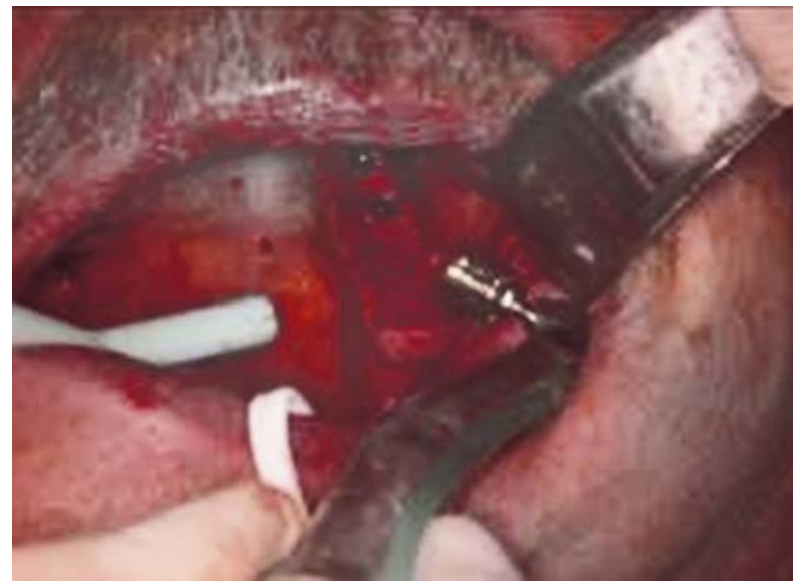

Fig. 5: Lateral sinus approach initiated with the dome drill and a $0.5 \mathrm{~mm}$ drill stopper 


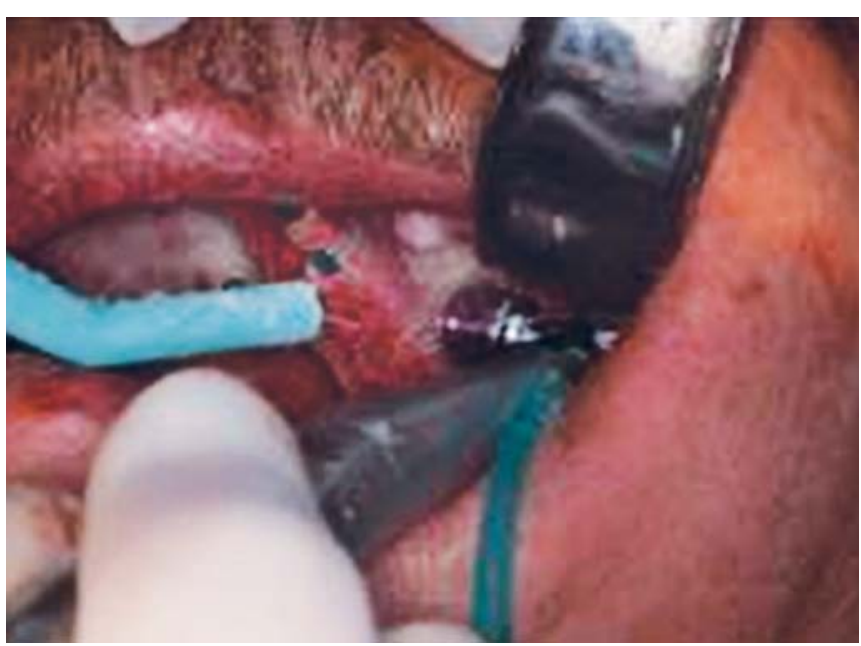

Fig. 6: Lateral sinus approach continued with the dome drill and a $1.0 \mathrm{~mm}$ drill stopper

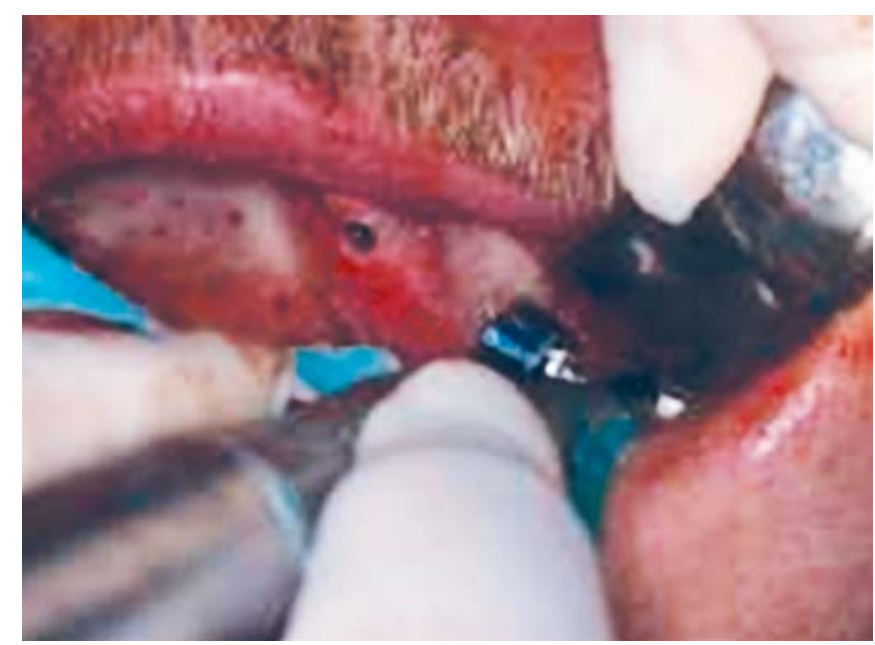

Fig. 7: Lateral sinus approach continued with the dome drill and a $1.5 \mathrm{~mm}$ drill stopper

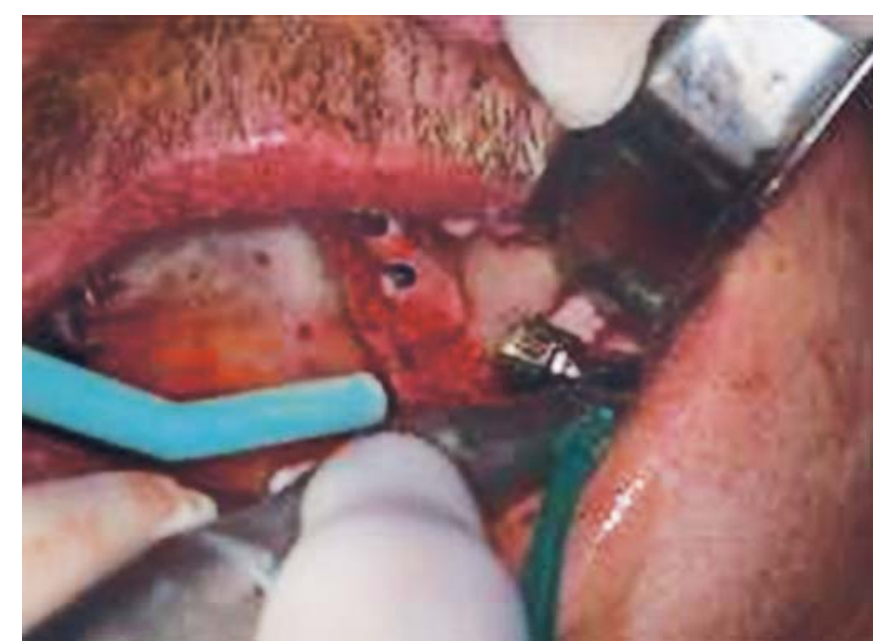

Fig. 8: Lateral sinus approach continued with the dome drill and a $2.0 \mathrm{~mm}$ drill stopper

(Figs 7 and 8). As bone is removed over the sinus membrane, the area changes in color from the light color of the bone (ivory) to a darker gray as the sinus membrane begins to

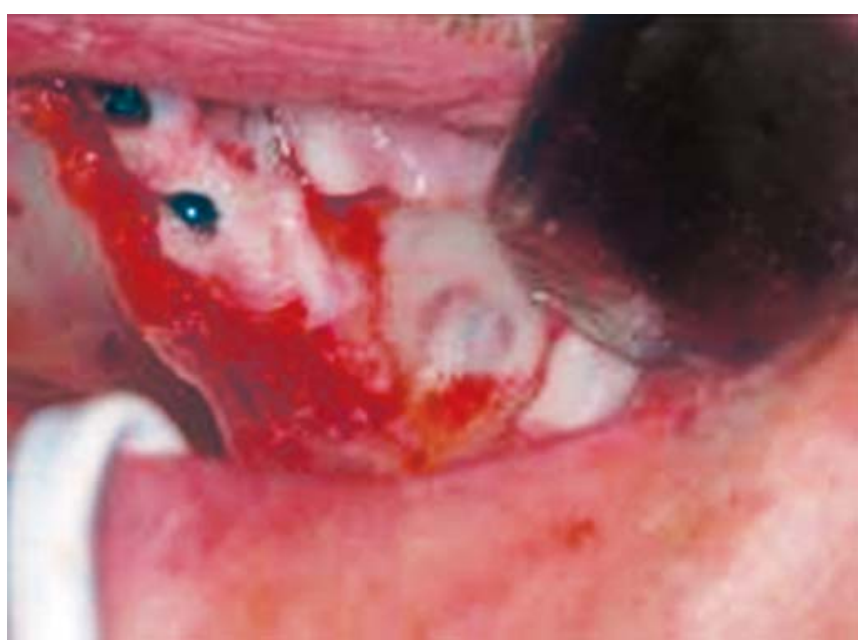

Fig. 9: Lateral wall of the maxillary sinus following sequential use of the dome drill with decreasing stopper depth demonstrating no damage to the sinus membrane after bone removal

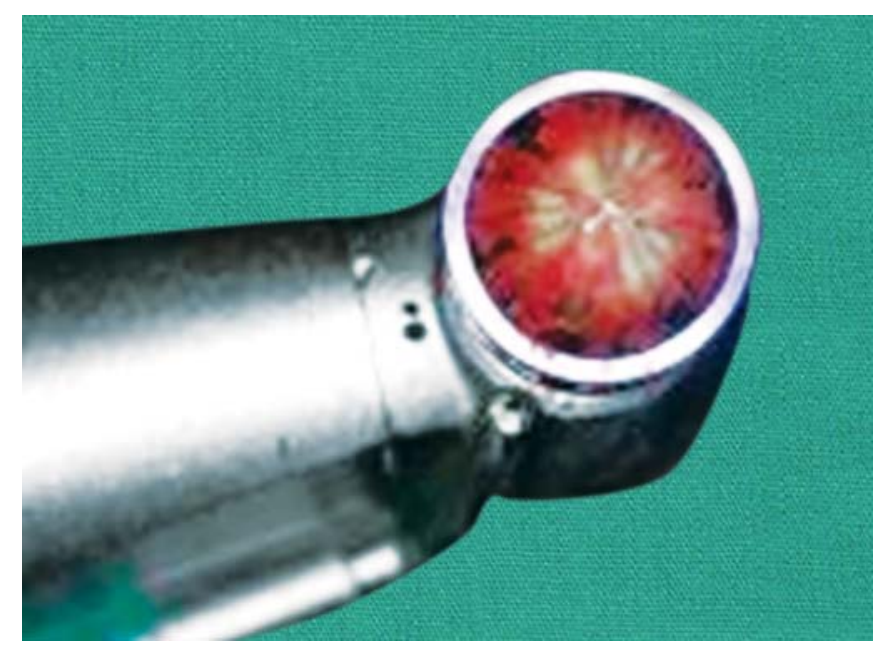

Fig. 10: Patient's bone collected in the dome drill that will be added to the osseous graft to be placed into the maxillary sinus

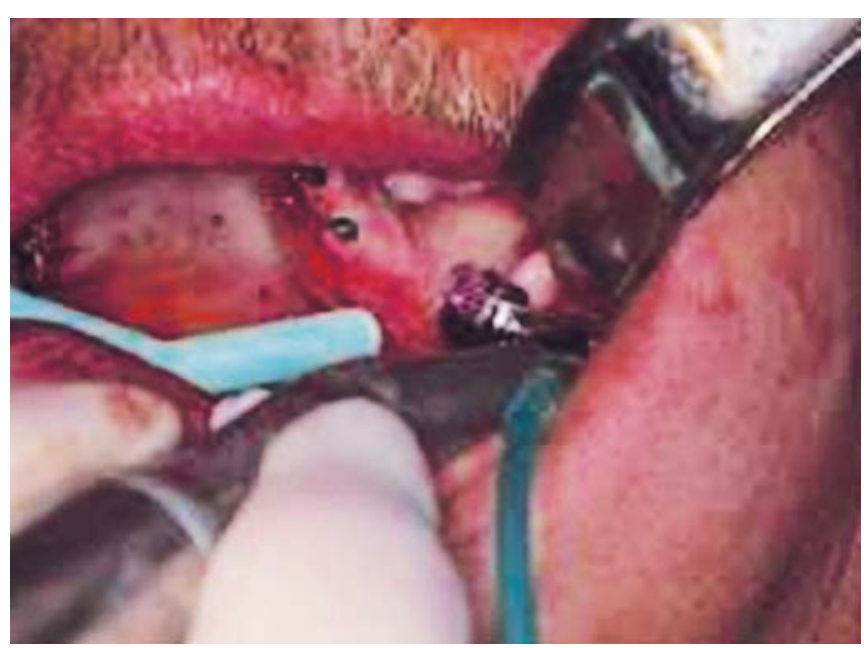

Fig. 11: Final dome drill with a $2.5 \mathrm{~mm}$ stopper utilized to fully expose the sinus membrane

show clinically at the window (Fig. 9). The osseous grinding from the dome drill is periodically removed from the drill to be used with the osseous graft to be placed (Fig. 10). 


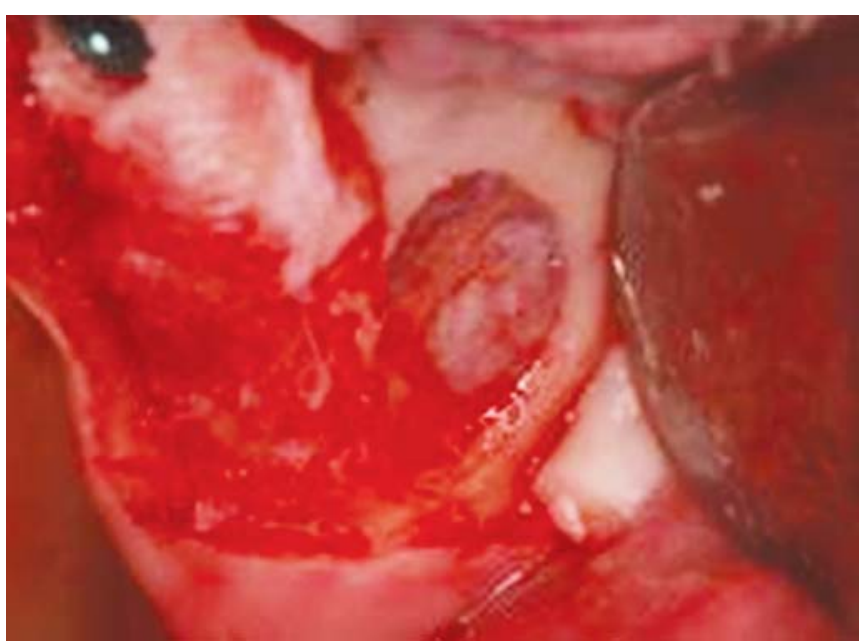

Fig. 12: Lateral window completed demonstrating the intact sinus membrane following use of the dome drills and stoppers. A bone septa is noted vertically in the window

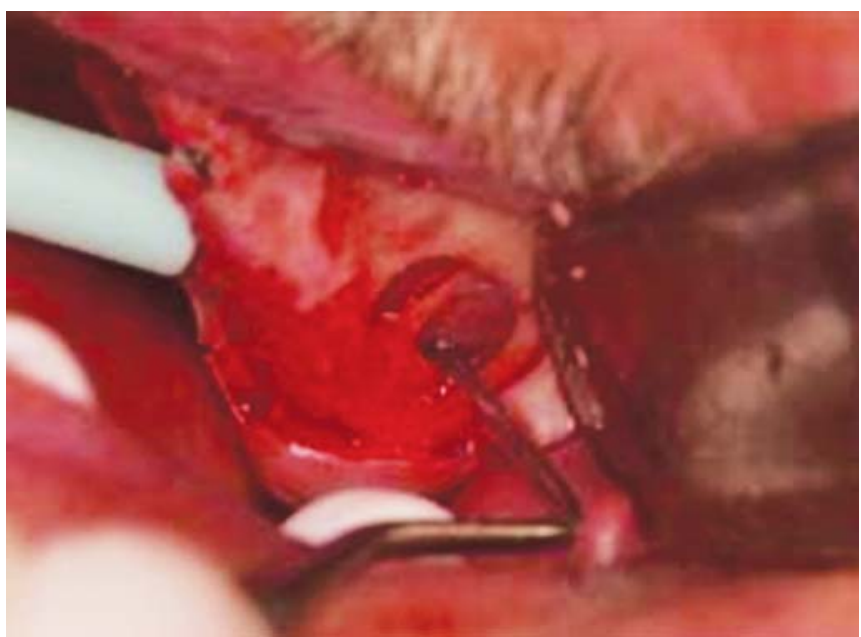

Fig. 13: Curettes utilized to tease the membrane from the bone wall and elevate the membrane in preparation for sinus grafting

Final window creation is made with the dome drill, in this particular case with a $2.5 \mathrm{~mm}$ drill stopper (Fig. 11). Some patients may require deeper drilling which is dependant on thickness of the lateral maxillary sinus wall. The intact sinus membrane is noted with no bone over the membrane at the window that has been created on the lateral wall (Fig. 12). An osseous septum is noted running vertically at the middle of the window created. The presence of the septum would preclude use of the core drill as it would be very difficult to remove the widow core created without tearing the sinus membrane.

Sinus curettes are utilized to start the sinus membrane elevation at the inferior aspect, teasing the membrane from the osseous wall of the sinus interiorly (Fig. 13). As this case has a septum the anterior and posterior aspects of the sinus in relation to the septum are elevated separately, with attention to teasing the membrane from the septum as part

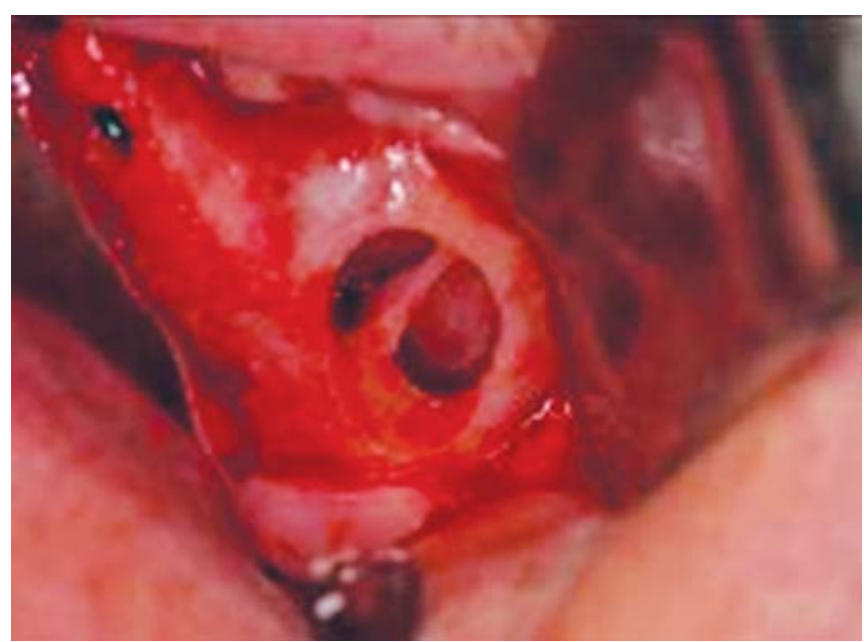

Fig. 14: Sinus membrane has been elevated and the bone septa is clearly noted

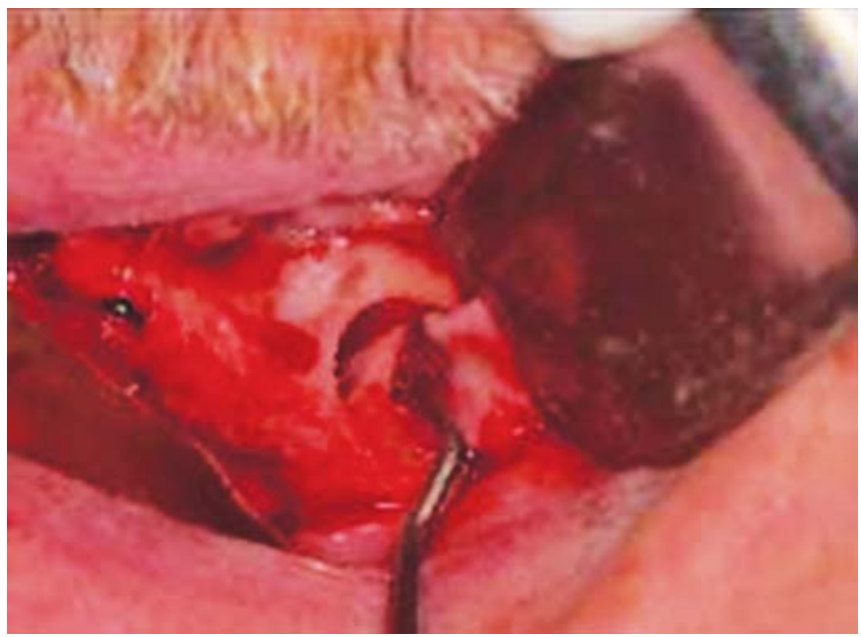

Fig. 15: Resorbable collagen tape an absorbable membrane (Ace surgical) is placed into the sinus to line the elevated membrane and assist in containing the osseous graft that will be placed

of the elevation process. It is important that the elevation also include the medial wall of the sinus so that fills a volume great enough that the implant when placed will be surrounded by bone (Fig. 14). Failure to elevate the medial wall aspect may result in the implant when placed having no osseous contact which may decrease implant success following loading. Additionally, the authors advise elevation to a greater height than the implant length to be placed when a delayed fixture placement is to be performed. This will allow for possible graft resorption during healing that may yield less height then was planned.

An absorbable collagen membrane (Resorbable Collagen Tape, Ace Surgical, Brockton, MA) is inserted into the sinus to act as a membrane to thicken the sinus membrane and seal any micro tears that might be present (Fig. 15). The Resorbable Collagen Tape is cut to size and placed into the sinus dry using the patient's blood in the 


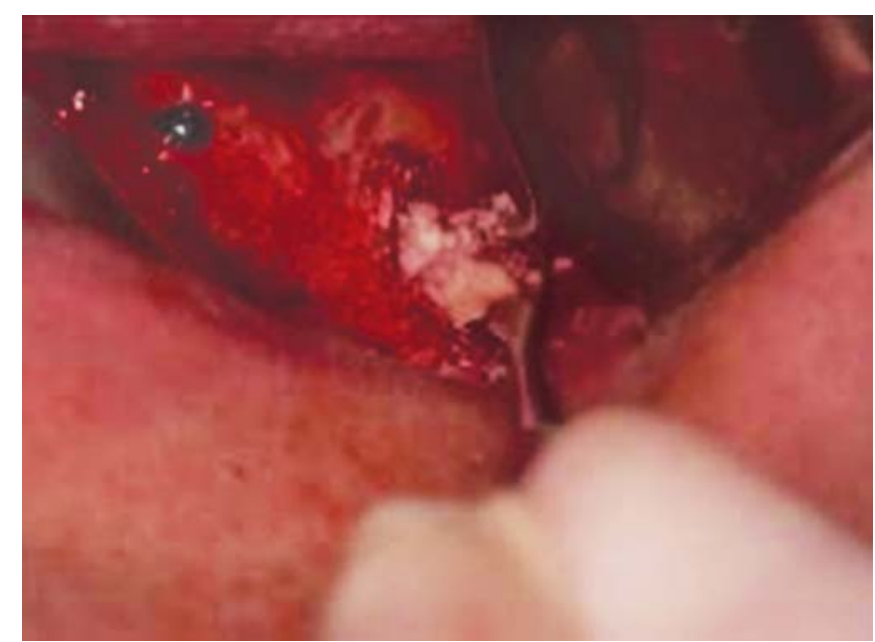

Fig. 16: Regenaform cortical cancellous bone chips (Exactech) is gently packed into the cavity created with sinus membrane elevation

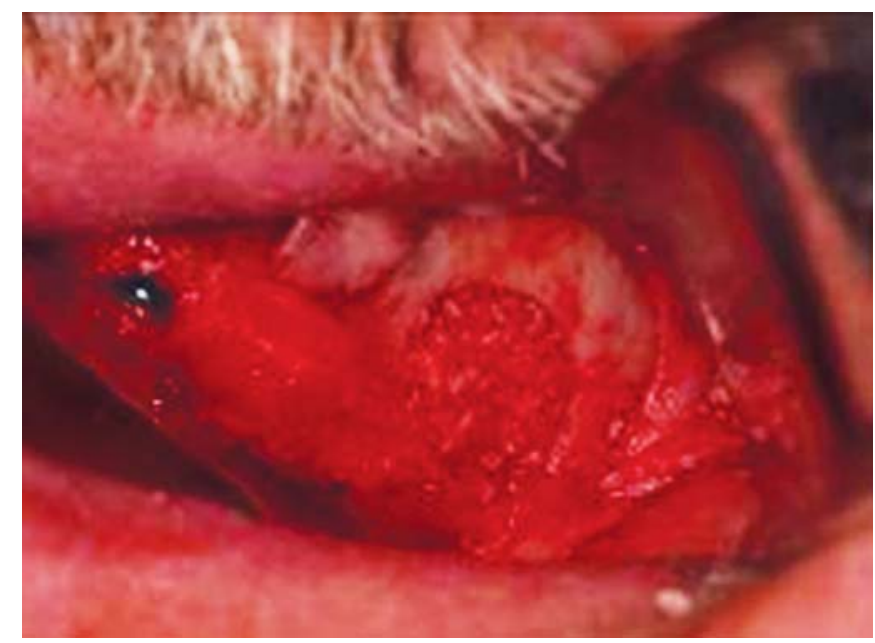

Fig. 17: Maxillary sinus has been filled with the Regenaform cortical cancellous bone chips (Exactech) to a level flush with the exterior lateral wall

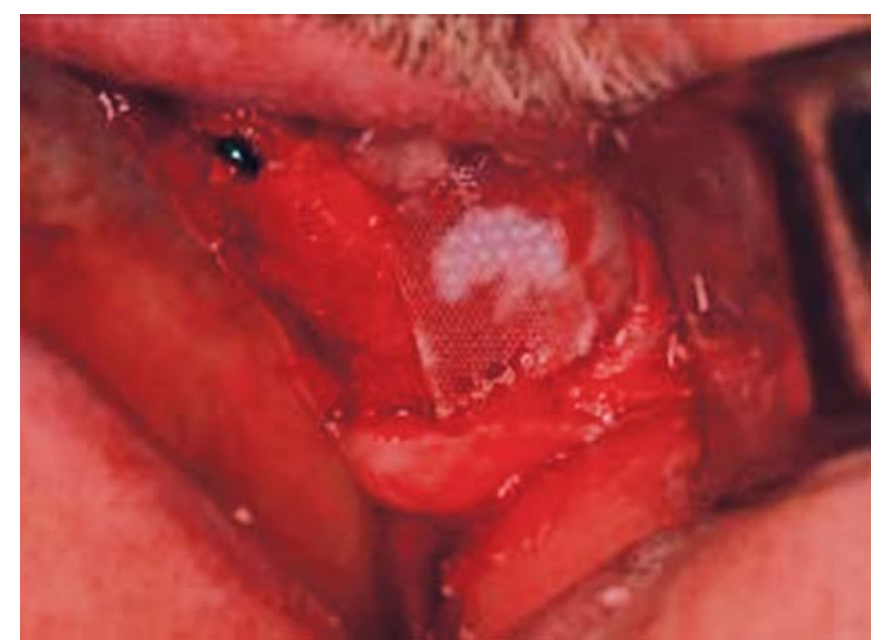

Fig. 18: Guidor, a resorbable membrane (Sunstar) is placed over the lateral window to prevent soft tissue in growth during graft organization

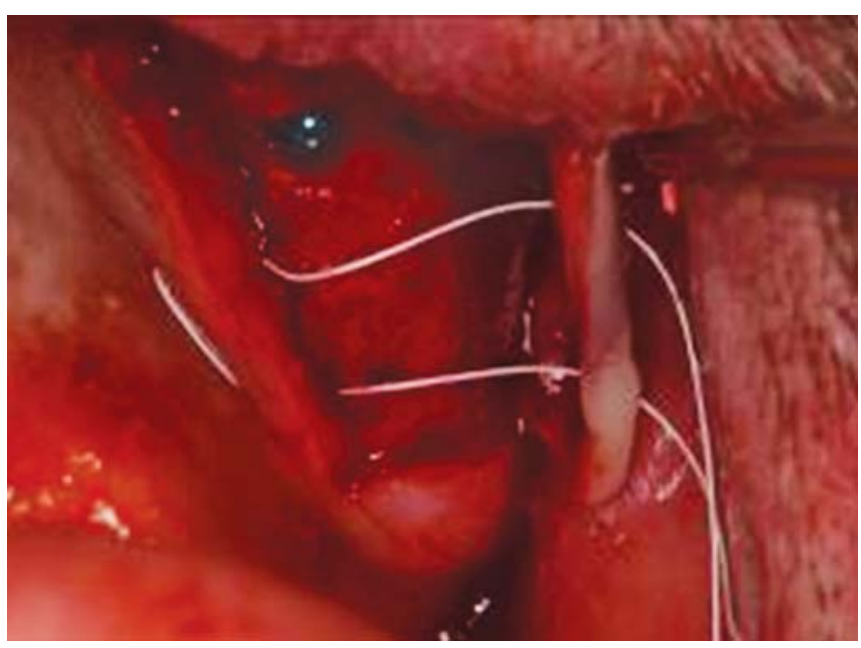

Fig. 19: Cytoplast PTFE suture (Osteogenics Biomedical) placed in a horizontal mattress technique is placed to close the flap with primary closure the goal

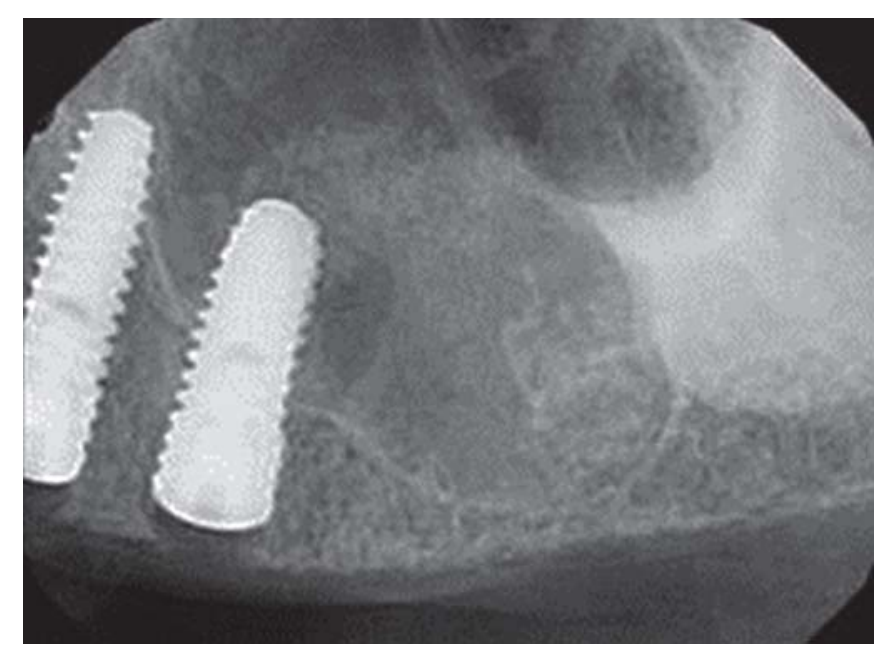

Fig. 20: Radiograph immediately after sinus augmentation demonstrating the new osseous height achieved with sinus elevation and grafting

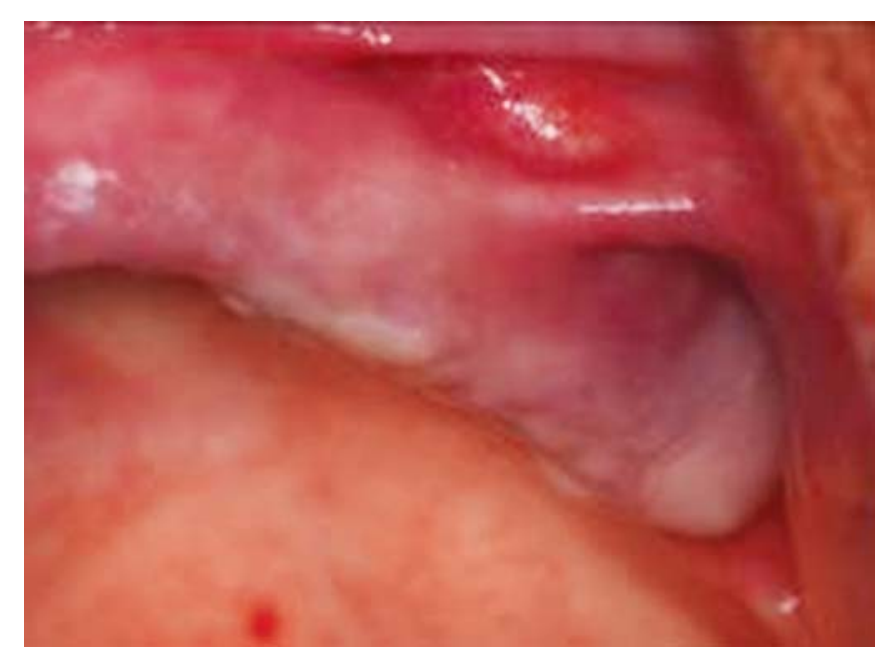

Fig. 21: Six months following sinus grafting the soft tissue shows no inflammation and is ready for implant placement into the grafted sinus 
site to wet it as it is placed. Once wetted with blood the Resorbable Collagen Tape becomes sticky attaching itself to the sinus membrane.

Autogenous bone collected from the dome drill was mixed with Regenaform cortical cancellous bone chips (Exactech, Gainesville, FL) in a sterile dappen dish and mixed. The osseous graft mixture was carried to the oral cavity and introduced into the elevated sinus and gently condensed with a large plugger, pushing the mixture to the medial and filling in a lateral direction until the entire cavity was filled (Fig. 16). The process was repeated in the cavity anterior to the septa. Sufficient osseous graft was placed till the sinus was augmented to be flush with the outer aspect of the lateral sinus wall at the window that had been created (Fig. 17).

A long-term resorbable membrane (Guidor, Sunstar, Chicago, IL) was cut to extend beyond the outline of the lateral window and placed over the osseous graft that had been placed into the sinus (Fig. 18). The flap was repositioned and initially closed with a horizontal mattress suture using Cytoplast ePTFE, (Osteogenics Biomedical, Lubbock, Texas) to achieve primary closure at the crest (Fig. 19). This suture serves to resist soft tissue tension that may result due to inflammation and the resulting swelling following surgery. Additional sutures are placed to close
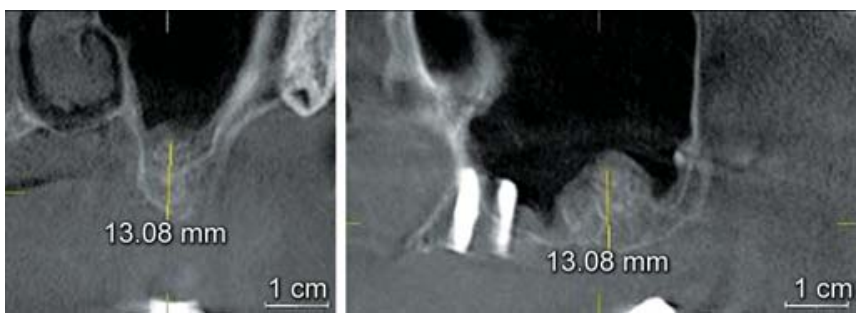

Fig. 22: Radiograph 6 months after sinus augmentation demonstrating the new osseous height achieved with sinus elevation and grafting

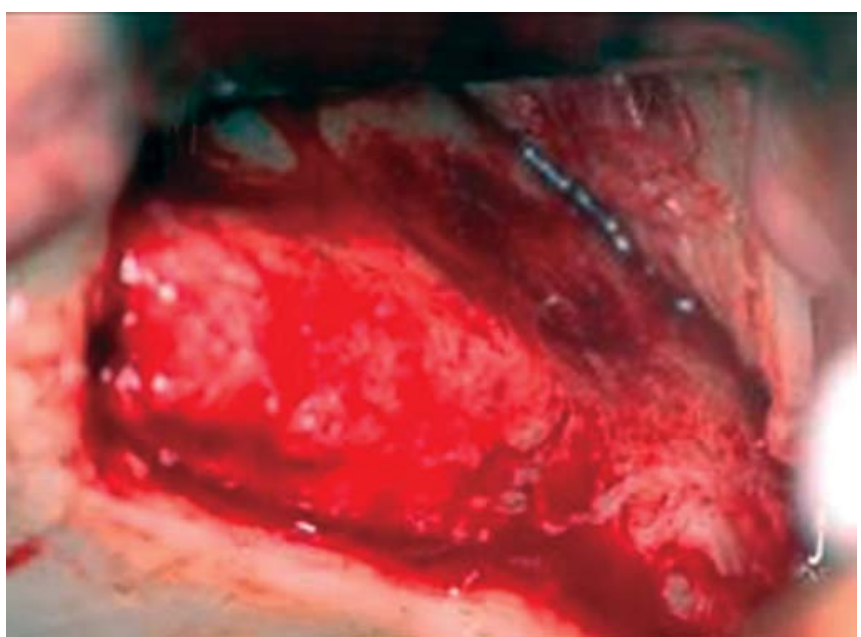

Fig. 23: The grafted site 6 months following sinus augmentation after full thickness flap elevation in preparation for implant placement the incision line using a simple interrupted technique. The radiograph shows initial graft placement and the elevation achieved to create a site that can accommodate implant placement at a later date (Fig. 20).

The patient returned 6 months following sinus augmentation for implant placement. Soft tissue in the site on the lateral aspect demonstrated no inflammation and incision lines were not discernable on the gingiva (Fig. 21). A radiograph was taken to check and verify the organization of the osseous graft that had been placed into the sinus and determine if the site was ready for re-entry for implant fixture placement (Fig. 22). A full thickness flap was made and the lateral aspect of the maxillary sinus where the window had been created demonstrating good integration of the graft with the surrounding native bone of the patient (Fig. 23).

\section{CONCLUSION}

Emphasis has moved to the use of a crestal approach to sinus elevation when additional osseous height is required for implant placement. This approach works well when at least $5 \mathrm{~mm}$ of osseous height is present for immediate implant placement. Yet when less bone height is present a lateral window approach may be a more prudent technique to increase crestal height so that implant fixtures may be placed.

The lateral sinus augmentation approach can be challenging as tearing of the sinus membrane often necessitates abandoning the procedure and re-entry at a later date after the membrane has healed. Previous techniques involved use of diamonds or carbides in a high speed handpiece to use of piezo surgical units. These approaches had potential for membrane damage (burs in a high speed) or were very slow (piezo). The LAS-Kit, from Hiossen/ Osstem utilizes special designed drills that greatly minimize tearing of the membrane and improve the safety of the procedure.

\section{REFERENCES}

1. Blomqvist JE, Alberius P, Isaksson S. Two maxillary sinus reconstruction with endosseous implants: a prospective study. Int J Oral Maxillofac implants 1998 Nov-Dec;13(6):758-766.

2. Valentini P, Abensur DJ. Maxillary sinus grafting with anorganic bovine bone: a clinical report of long-term results. Int J Oral Maxillofac Implants 2003 Jul-Aug;18(4):556-560.

3. Tong DC, Drangsholt M, Beirne OR. A review of survival rates for implants placed in grafted maxillary sinuses using metaanalysis. Int J Oral Maxillofac Implants 1998 MarApr;13(2):175-182.

4. Tatum OH Jr. Maxillary and sinus implant reconstructions. Dent Clin North Am 1986 Apr;30(2):207-229.

5. Rosen PS, Summers R, Mellado JR, Salkin LM, Shanaman RH, Marks MH, Fugazzotto PA. The bone-added osteotome sinus 
floor elevation technique: multicenter retrospective report of consecutively treated patients. Int J Oral Maxillofac implants 1999 Nov-Dec;14(6):853-858.

6. Summers RB. A new concept in maxillary implant surgery: the osteotome technique. Compend Contin Educ Dent 1994 Feb;15(2):152-162.

7. Summers RB. The osteotome technique: part 3-less invasive methods of elevating the sinus floor. Compend Contin Educ Dent 1994 Jun;15(6):698-710.

8. Emmerich D, Att W, Stappert C. Sinus floor elevation using osteotomes: a systemic review and meta-analysis. J periodontal 2005 Aug;76(8):1237-1251.

9. Tofflen M. Osteotome-mediated sinus floor elevation: a clinical report. Int J Oral Maxillofac Implants 2004 Mar-Apr;19(2): 266-273.

10. Peleg M, Mazor Z, Chaushu G, Garg AK. Sinus floor augmentation with simultaneous implant placement in the severely atrophic maxilla. J Periodontal 1998 Dec;69(12): 1397-1403.

11. Peleg M, Mazor Z, Garg AK. Augmentation grafting of the maxillary sinus and simultaneous implant placement in patients with 3 to $5 \mathrm{~mm}$ of residual alveolar bone height. Int J Oral Maxillofac Implants 1999 Jul-Aug;14(4):549-556.

\section{ABOUT THE AUTHORS \\ Douglas F Dompkowski (Corresponding Author)}

Private Practitioner, Periodontology and Implant Dentistry, Bethesda Maryland; Faculty, Department of Periodontology, University of Maryland, Maryland, USA, e-mail: dompkowskidds@verizon.net

\section{Gregori M Kurtzman}

Private General Practice, Silver Spring, Maryland, USA 